\title{
Origin of Star-to-Star Abundance Inhomogeneities in Star Clusters
}

\author{
Jan Palouš ${ }^{1}$, Richard Wünsch ${ }^{2}$, \\ Guillermo Tenorio-Tagle ${ }^{3}$, and Sergyi Silich ${ }^{3}$ \\ ${ }^{1}$ Astronomical Institute, Academy of Sciences of the Czech Republic, \\ Boční 1401, 14031 Prague 4, Czech Republic \\ email: palous@ig.cas.cz \\ ${ }^{2}$ Cardiff University, Queens Buildings, The Parade, Cardiff, \\ CF24 3AA, United Kingdom \\ email: richard@wunsch.cz \\ ${ }^{3}$ Instituto Nacional de Astrofísica Optica y Electronica, \\ AP 51, 72000 Puebla, México \\ email: gtt@inaoep.mx, silich@inaoep.mx
}

\begin{abstract}
The mass reinserted by young stars of an emerging massive compact cluster shows a bimodal hydrodynamic behaviour. In the inner part of the cluster, it is thermally unstable, while in its outer parts it forms an out-blowing wind. The chemical homogeneity/inhomogeneity of low/high mass clusters demonstrates the relevance of this solution to the presence of single/multiple stellar populations. We show the consequences that the thermal instability of the reinserted mass has to the galactic super-winds.
\end{abstract}

Keywords. Galaxy: abundances, Galaxy: Globular clusters: general, Galaxy: open star clusters and associations: general, galaxies: star clusters, galaxies: starburst

\section{Introduction}

The open star clusters and stellar moving groups have internally homogeneous chemical composition. Clusters like the Hyades, Collinder 261, the Herculis stream or the moving group HR 1614 are chemically unique, distinguishable one from the other, showing no pollution from secondary star formation (De Silva et al., 2008). The chemical homogeneity of open star clusters like the Hyades (De Silva et al., 2006) and Collinder 261 (De Silva et al., 2007) proves that they have been formed out of a well-mixed cloud and that any self-enrichment of stars did not take place there.

Young and massive stellar clusters, frequently called super star clusters, are preferentially observed in interacting galaxies. Their stellar mass amounts to several million $\mathrm{M}_{\odot}$ within a region less than a few parsecs in diameter. They represent the dominant mode of star formation in starburst galaxies. Their high stellar densities resemble those of globular clusters, where several stellar populations have been observed (Piotto, 2008).

To explain the presence of multiple stellar generations in globular clusters, the slow wind emerging from a first generation of fast rotating massive stars is invoked by Decressin et al. (2007). (See also the review by Meynet (2008) in this volume.) The authors argue that the fast rotating massive stars function as a filter separating the H-burning products from later products of He-burning. However, it is not clear why all the massive stars rotate fast, or why the slow wind produced by stellar rotation is just retained inside the potential well of the stellar cluster.

An alternative solution, how to form the second generation of stars in massive star clusters, is proposed in models of star cluster winds described by Tenorio-Tagle et al. 
(2007), Wünsch et al. (2007), and Wünsch et al. (2008). There, we argue that a critical mass of a cluster exists, below which the single-mode hydrodynamical solution to the cluster winds applies. Such clusters should have one stellar generation only, and show strong winds corresponding to the momentum and energy feedback of all their stars. The clusters above the critical mass should follow the bi-modal solution to their winds, where only the outer skin of the cluster participates in the wind. Their inner parts are thermally unstable, and hence being the potential places of secondary star formation.

\section{The Model}

The hydrodynamical behaviour of matter reinserted within a star cluster is described by Chevalier \& Clegg (1985). In this adiabatic model, the authors assume that all the energy provided by stellar winds and supernovae is thermalized in random collisions of the shock waves creating a gas of temperatures $T>10^{7} \mathrm{~K}$. Chevalier \& Clegg's stable solution shows almost constant density and temperature inside of the cluster. A mild outward pressure gradient drives a cluster wind with a radially increasing velocity reaching the sound speed $c_{S C}$ at the cluster surface and approaching $2 c_{S C}$ at infinity. The run of wind particle density, temperature and wind velocity in this single-mode model is
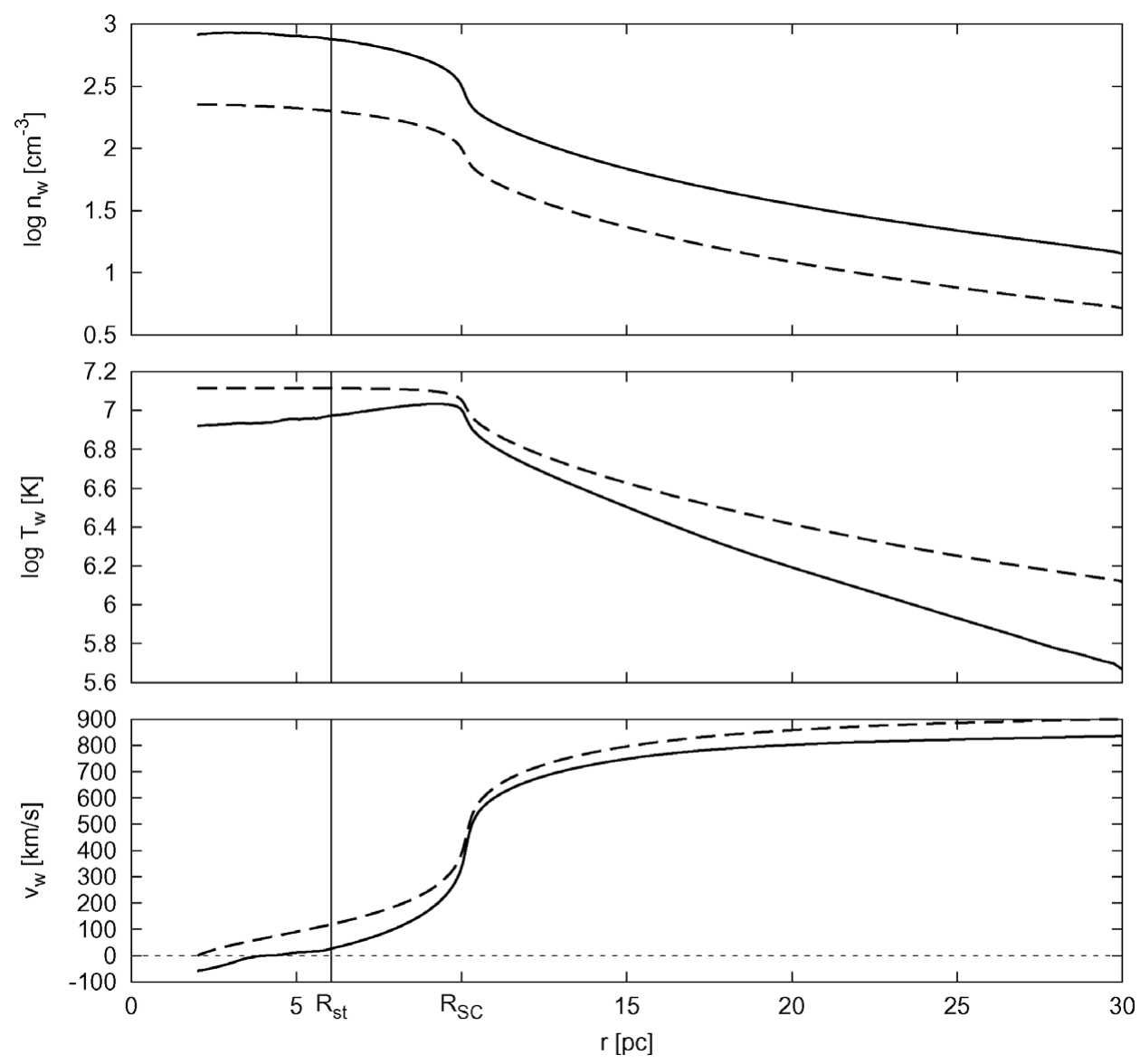

Figure 1. Radial profiles of particle density, temperature and velocity for single-modal (dashed lines) and bi-modal (solid lines) solutions. 

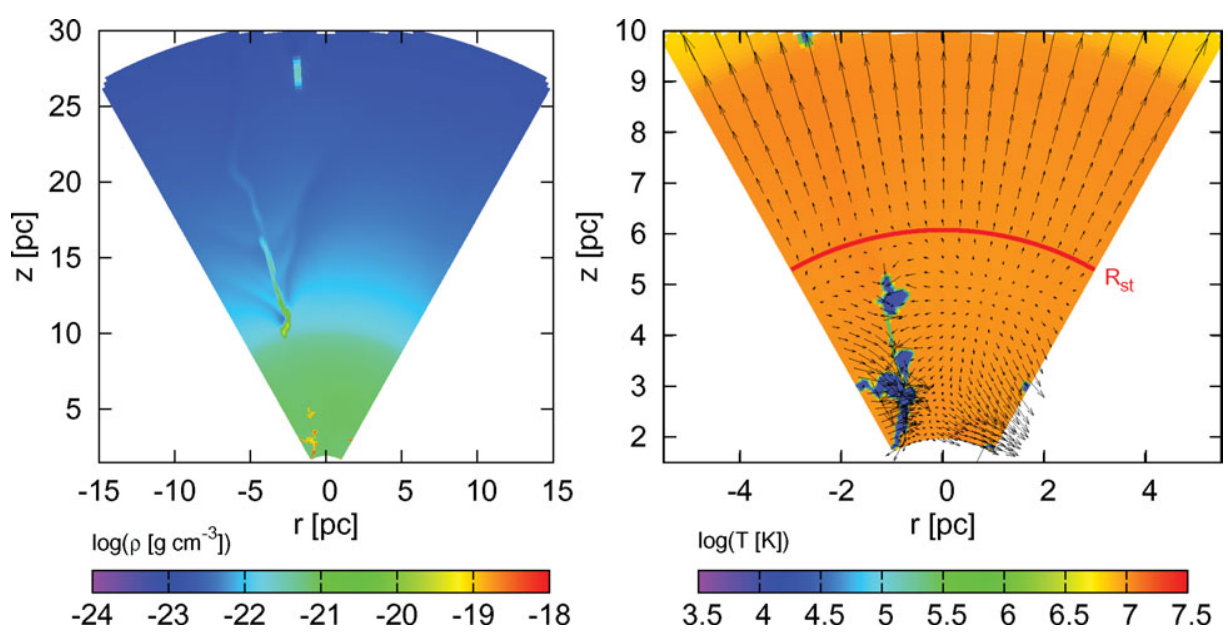

Figure 2. Bi-modal solution: density distribution (left panel), temperature distribution with velocity vectors (right panel) $0.56 \mathrm{Myr}$ after the beginning of the simulation.

shown in Fig. 1. With the adiabatic model the more massive clusters produce the more powerful wind, taking away all the elements produced in stars. Thus, any inhomogeneity in stellar chemical composition reflects the abundance distribution in the parental starforming nebula. If the original cloud is well mixed, according to the adiabatic, single-mode model of Chevalier \& Clegg (1985), the star cluster will have one population of the same chemical composition.

The assumption that all the mechanical energy of winds and supernovae is thermalized need not to be completely true. The efficiency of the thermalization process $\eta$ depends on the details of shock-shock collisions and different authors give different values ranging from 0.01 to 1 (Melioli, \& Gouveia Dal Pino, 2004). The value of $\eta$ may depend on Mach numbers of the colliding shocks and/or on the chemical composition of the colliding fronts regulating what part of the mechanical energy is directly radiated away. The value of $\eta$ may be different in the case of colliding stellar winds, or colliding supernova explosions.

The density of the cluster wind $n_{w}$ depends on the stellar density: for a cluster of given radius $R_{S C}, n_{w}$ is proportional to the cluster mass $M_{S C}$. On the other hand, the radiative cooling rate is proportional to $n_{w}^{2}$ multiplied with a cooling function $\Lambda(T, Z)$, where $Z$ stands for the chemical composition of the radiating plasma. Thus, the radiative cooling rate is proportional to $M_{S C}^{2}$. However, the cluster mechanical luminosity $L_{S C}$, or the part that is thermalized $\eta L_{S C}$, depends linearly on cluster mass $M_{S C}$, which implies that a critical mass $M_{S C \text {, crit }}$ must exist, above which the energy loss due to radiative cooling and gas expansion exceeds $\eta L_{S C}$. Its value depends on $R_{S C}$ and $\eta$.

Less massive clusters show the single-mode behaviour. They take away at least a part of the mechanical luminosity as winds from all the cluster volume. In the adiabatic approximation, which applies to low mass clusters far below the $M_{S C, \text { crit }}$, all the mechanical energy, which is transformed to heat, is removed as winds.

For $M_{S C}$ above the critical value, the volume inside of the cluster is split into two sectors. The inner part of the cluster, inside the stagnation radius $R_{S T}$, is thermally unstable. There, the instability leads to fast cooling of small parcels of gas surrounded by a hot medium. The repressurizing shocks drive the hot gas into cold gas parcels forming high density gas concentrations. The outer part of the cluster, its skin $R_{S T}<R<R_{S C}$, shows the out-blowing wind. The run of wind particle density, temperature and wind 
velocity in the bi-modal solution of the star cluster wind is shown in Fig. 1. The singlemode compared to the bi-modal solution shows wind of lower density and higher temperature. The velocity of the out-blowing wind is higher in the case of the single-mode solution compared to the bi-modal case; the two solutions have the same velocity at the cluster surface only. 2D hydrodynamical simulation of cluster winds are described by Wünsch et al. (2008). In Fig. 2 we show the density and temperature distribution together with velocity vectors for a star cluster with $R_{S C}=10 \mathrm{pc}, \eta=1$, and $L_{S C}=$ $10^{42} \mathrm{erg} \mathrm{s}^{-1}$. We can clearly distinguish the thermally unstable region within the stagnation radius $R_{S T}=6 \mathrm{pc}$, and the wind blowing from the outer skin of the cluster. The dense concentration, in the thermally unstable part of the cluster, shows a small velocity dispersion relative to the cluster; most of these concentrations are unable to leave the cluster. Thus they accumulate in the inner volume, becoming potential places of secondary star formation.

\section{Summary}

We propose a bimodal solution, where in the central part of a massive star cluster, a thermally unstable region forms (see Fig. 2). In this region, the thermal instability creates cold regions surrounded by hot medium imploding into them. The high-velocity wing of broad spectral lines Gilbert, \& Graham (2007) observed in SSC may be created by imploding shock in the vicinity of thermally unstable parcels of gas.

The second generation of stars may be formed out of cold clumps produced by thermal instability. During the early evolution of a massive cluster, the first Myrs, the mechanical energy input is dominated by stellar winds (Leitherer et al. 1999). The efficiency of thermalization $\eta$ may be low in this case, and a massive cluster may be above $M_{S C, \text {, rit }}$, since its value is low. Later, the importance of winds fades out, and the mechanical input is dominated by supernovae. This may increase the thermalization efficiency $\eta$, increasing at the same time the value $M_{S C, \text { crit }}$. Thus the same massive cluster, which was initially in the bi-modal situation moves to single-mode situation.

The cold parcels of gas form, in the cluster central part during the early bi-modal situation, from the winds that are enriched by products of H-burning. The later Heburning products are inserted into the cluster volume when the mechanical energy input is dominated by supernovae, which may mean that the cluster is in the single-mode situation, and the wind clears its volume from He burning products. Thus, the thermal instability, which operates during a few initial Myr in the central part of the cluster, may produce a second generation of stars enriched by H-burning products. Later, the cluster moves into the single-mode situation, which means that it is able to expel the He-burning products.

The feedback of massive stars in super star clusters creates galactic winds, or super winds, reaching to large distances from the parent galaxies, transporting the products of stellar burning into intergalactic space. The bimodal solution, providing a possible explanation of multiple stellar populations in globular clusters, limits the super winds. During the initial period of cluster evolution, when the stellar winds dominate the mechanical energy input, the super wind is restricted only to the outer skin of the cluster, which means that it is rather weak. Only later, when supernovae become dominant in mechanical energy input, strong super winds blowing out from all the cluster volume may reach large distances from their parent galaxies. How effective the super winds of super star clusters can be in transporting the products of stellar evolution into intergalactic spaces should be discussed in future. 


\section{Acknowledgements}

The authors gratefully acknowledge the support by the Institutional Research Plan AV0Z10030501 of the Academy of Sciences of the Czech Republic and by the project LC06014 Center for Theoretical Astrophysics of the Ministry of Education, Youth and Sports of the Czech Republic. RW acknowledges support by the Human Resources and Mobility Programme of the European Community under the contract MEIF-CT-2006039802. This study has been supported by CONACYT-México research grant 60333 and 47534-F and AYA2004-08260-CO3-O1 from Spanish Consejo Superior de Investigaciones Científicas. The authors express their thanks to Jim Dale for careful reading of the text.

\section{References}

Chevalier, R. A. \& Clegg, A. W. 1985, Nature, 317, 44

De Silva, G. M., Sneden, C., Paulson, D. B., Asplund, M., \& Bland-Hawthorn, J. 2006, AJ, 131, 455

De Silva, G. M., Freeman, K. C., Asplund, M., Bland-Hawthorn, J., Bessesl, M. S., \& Collet, R. 2007, AJ, 133, 1161

De Silva, G. M., Freeman, K, Bland-Hawthorn, J., \& Asplund, M. 2008, in J. Andersen, J. Bland-Hawthorn \& B. Nordstöm (eds.), The Galaxy Disk in Cosmological Context, Proc. IAU Symposium No. 254 (CUP), this volume

Decresssin, T., Charbonnel, C., \& Meynet, G. 2007, A\&A, 475, 859

Gilbert, A. M. \& J. R. Graham. 2007, ApJ, 668, 168

Leitherer, C., Schaerer, D., Goldader, J. D., Gonzáles-Delgado, R. M., Robert, C., Foo Kune, D., De Mello, D. F., Devost, D., \& Heckman, T. M. 1999, ApJS, 123, 3

Melioli, C. \& de Gouveia Dal Pino, E. M. 2004, A\& $A$, 424, 817

Meynet, G. 2008, in J. Andersen, J. Bland-Hawthorn \& B. Nordstöm (eds.), The Galaxy Disk in Cosmological Context, Proc. IAU Symposium No. 254 (CUP), this volume

Piotto, G. 2008, MemSAI, 79, 3

Tenorio-Tagle, G., Wünsch, R., Silich, S., \& Palouš, J. 2007, ApJ, 658, 1196

Wünsch, R., Silich, S., Palouš, J., \& Tenorio-Tagle, G. 2007, A\&A, 471, 579

Wünsch, R., Tenorio-Tagle, G., Palouš, J., \& Silich, S. 2008, ApJ, 684, September 1, arXiv:0805.1380v1 


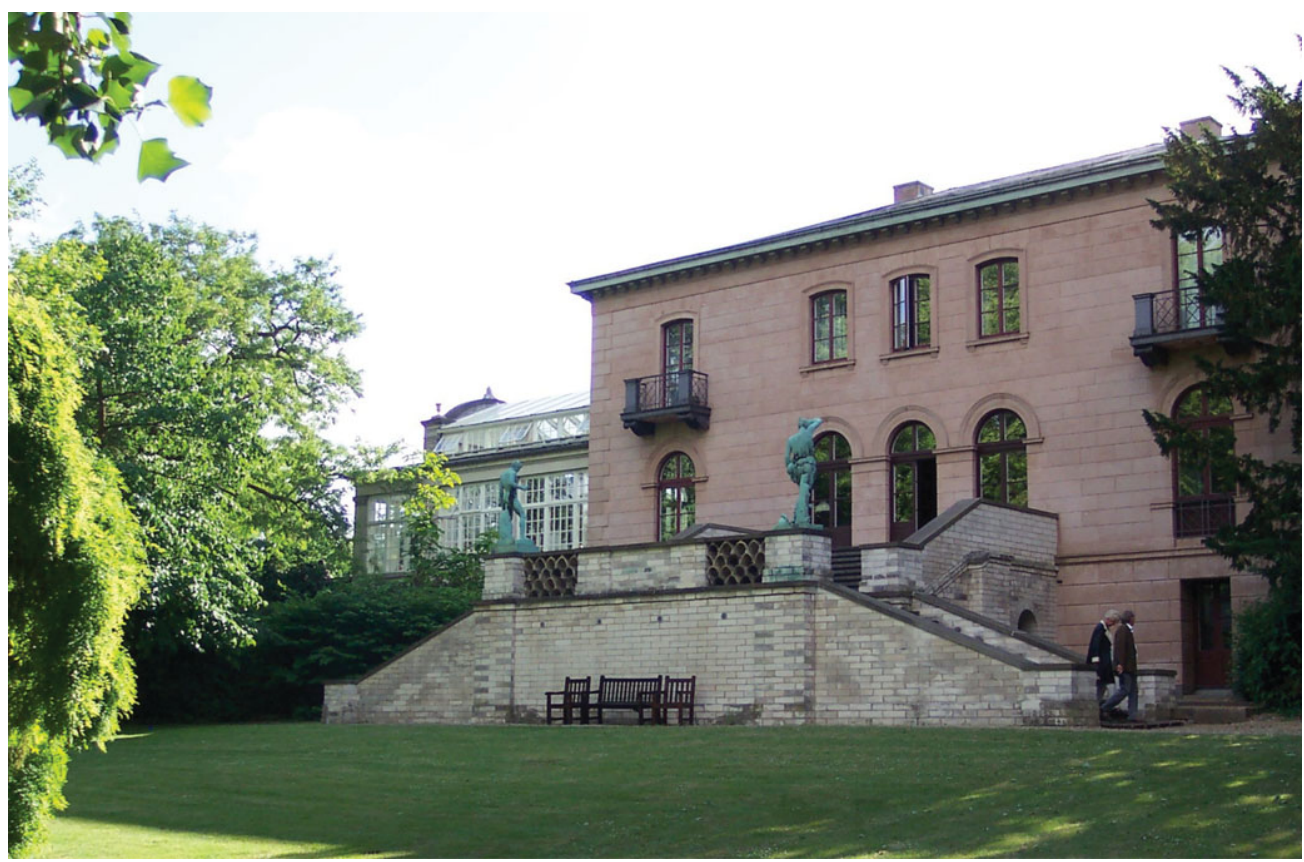

The former Strömgren residence of honour, seen from the park.

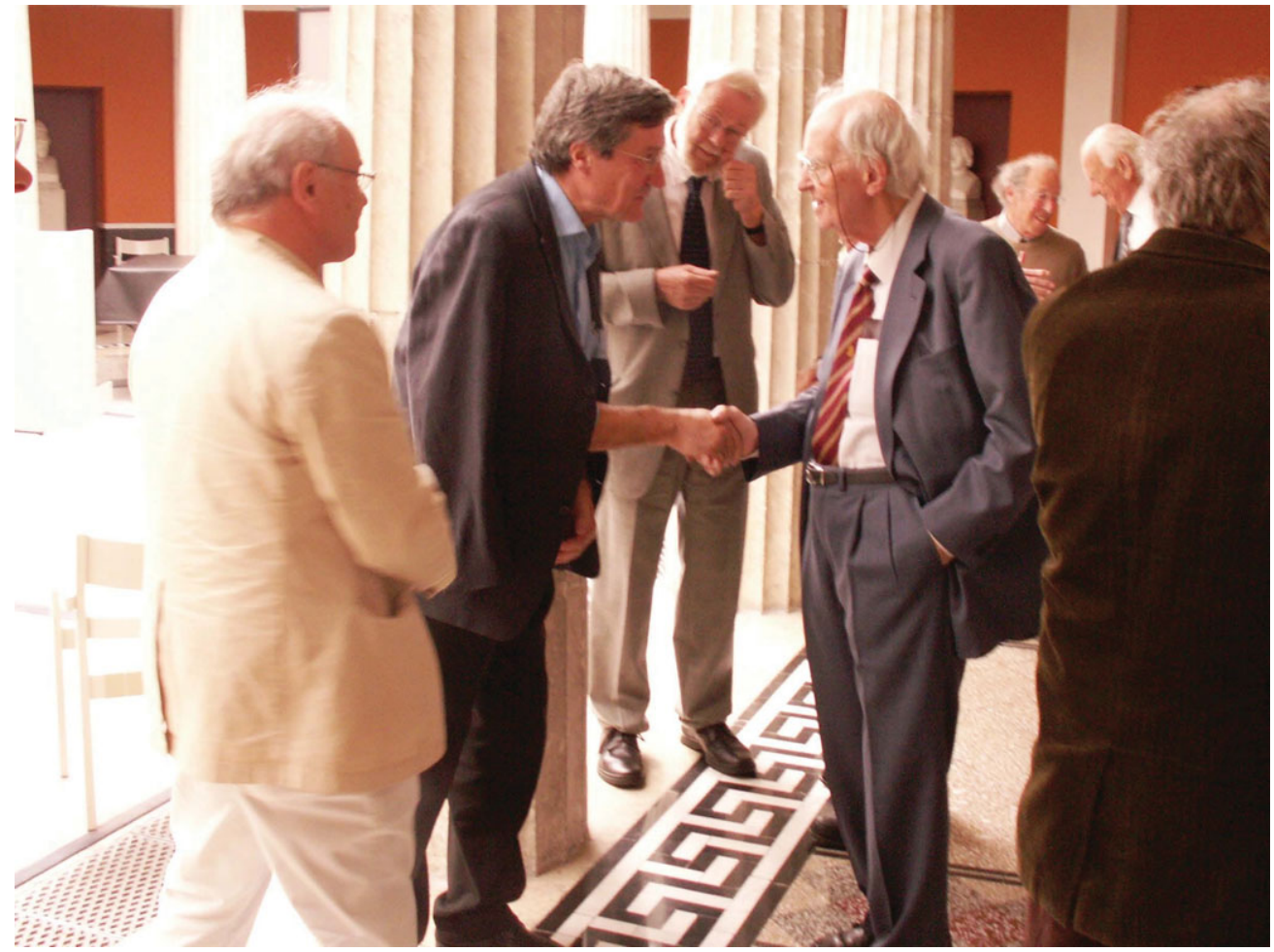

Jan Palouš meets Adriaan Blaauw at Carlsberg, with Hans Zinnecker (left). In the background, Ole Strömgren, Ben Mottelson, and Haldor Topsøe (photo: Bruce Elmegreen). 